\title{
PROGNOSTIC VALUE OF TUMOR STROMA RATIO IN TRIPLE NEGATIVE BREAST CANCER
}

DOI: 10.36740/WLek202103201

\author{
Liubov M. Zakhartseva ${ }^{1,2}$, Mariia A. Yanovytska ${ }^{1,2}$ \\ 'KYIV CITY CLINICAL ONCOLOGY HOSPITAL, KYIV, UKRAINE \\ 2BOGOMOLETS NATIONAL MEDICAL UNIVERSITY, KYIV, UKRAINE
}

\begin{abstract}
The aim: The purpose of this study is to investigate prognostic value of tumor stroma ratio in triple negative breast carcinomas.

Materials and methods: This cohort retrospective study included a total number of 232 previously untreated operational materials with primary stage I-III triple negative breast cancer. The median follow-up period was 3.8 years for overall survival and 3.2 years for disease-free survival. Tumor stroma ratio was evaluated by two pathologists (Kappa coefficient was 0.71 and 0.84 , respectively).

Results: Kaplan-Meier curves with logrank test statistically significantly showed relationship between tumor stroma ratio and both overall and disease-free survival. The Cox proportional hazards model showed tumor stroma ratio is a strong independent prognostic factor for triple negative breast carcinomas with hazard ratios of 2.11 ( $p=0.002)$ for overall survival and $1.83(\mathrm{p}=0.004)$ for disease-free survival in multivariate analysis.

Conclusions: Triple negative breast tumors with high stroma ratio have worse overall and disease-free survival compared to low stroma ratio tumors. Investigation of tumor stroma ratio doesn't require any additional costs and slide preparation. It can be added to routine breast cancer investigation to expand knowledge about cancer prognosis.
\end{abstract}

KEY WORDS: triple negative breast cancer, tumor microenvironment, stromal cells, cancer associated fibroblasts

Wiad Lek. 2021;74(3 p.II):565-571

\section{INTRODUCTION}

Breast cancer remains to be among leading women cancers worldwide. Triple negative breast cancer which lacks estrogen, progesterone and human epidermal growth factor receptor 2 (HER2) amplificationloverexpression has the worst outcomes compared to other molecular subgroups $[1,2]$. Despite the progress the treatment options for triple negative disease are still limited [3]. Questions about over- and under treatment have forced search of additional prognostic markers.

Interactions between tumor microenvironment (TME) and tumor itself play an important role in cancer progression, as well as in metastatic potential and chemotherapy resistance [4]. Tumor stroma ratio (TSR) which is a part of TME is of increased investigation interest in this regard. It is a parameter which can be easily evaluated on $\mathrm{H} \& \mathrm{E}$ slide. It doesn't require additional slides and takes minimal time consume. TSP has already been investigated in cancers of different localizations, in particular tumors of gastrointestinal tract $[5,6]$ where it became evident TSR is an independent prognostic factor for survival. It was also investigated as a predictive marker in some other types of tumors $[7,8]$. Several research papers indicate that tumor stroma ratio is also an independent prognostic marker for breast cancer $[9,10]$ including triple negative cancer as well $[9,11]$. Gujam et al [12] found that high tumor stroma ratio was also statistically significantly associated with high tumor grade, lymph node positivity, low CD68 macrophage infiltrate and shorter cancer-specific survival. However, they stated that TSP was not an independent prognostic factor for triple negative patients. Kramer et al. [13] published a literature review on the prognostic value of TSR in breast cancer patients. General trend goes towards high stroma ratio and poor overall survival with more significant hazard ratios in triple negative tumors. Vangangelt et al. [14] showed that the TSR was most discriminative in triple-negative tumors and also in grade III tumors, compared to grade I and grade II. TSR was not modified by age, tumor size, histology, ER status, PR status, HER2 status and lymph node status which advices TSR as a potential prognostic factor.

\section{THE AIM}

In this research, we continue to expand the value of TSR as prognostic parameter for highly heterogeneous group of triple negative breast carcinomas.

\section{MATERIALS AND METHODS}

A total number of 350 samples from patients with triple negative breast cancer treated during 2009-2018 in Kiev City Oncology Hospital was investigated. Histopathological assessment was made only for previously untreated 
Table 1. General characteristics of histological parameters of tumors and treatment options.

\begin{tabular}{|c|c|c|}
\hline \multirow{2}{*}{ Parameter } & \multicolumn{2}{|c|}{ Number } \\
\hline & N (232) & $\%$ \\
\hline \multicolumn{3}{|l|}{ Age } \\
\hline$=<40$ & 22 & 9.5 \\
\hline$>40<60$ & 114 & 49.1 \\
\hline$>60$ & 96 & 41.4 \\
\hline \multicolumn{3}{|l|}{ Stage } \\
\hline 1 & 76 & 32.8 \\
\hline II & 124 & 53.4 \\
\hline III & 32 & 13.8 \\
\hline \multicolumn{3}{|l|}{ pT (Tumor) } \\
\hline $\mathrm{pT} 1$ & 91 & 39.2 \\
\hline pT2 & 129 & 55.6 \\
\hline рT3 & 5 & 2.2 \\
\hline pT4 & 7 & 3.0 \\
\hline \multicolumn{3}{|l|}{ pN (Nodes) } \\
\hline pNO & 173 & 74.6 \\
\hline $\mathrm{pN} 1$ & 34 & 14.6 \\
\hline $\mathrm{pN} 2$ & 15 & 6.5 \\
\hline pN3 & 10 & 4.3 \\
\hline \multicolumn{3}{|l|}{ Histologic type } \\
\hline NST (No special type) & 197 & 84.9 \\
\hline Lobular & 9 & 3.9 \\
\hline Papillary & 10 & 4.3 \\
\hline Medullary features & 9 & 3.9 \\
\hline Micropapillary & 1 & 0.4 \\
\hline Secretory & 2 & 0.9 \\
\hline Metaplastic & 1 & 0.4 \\
\hline Adenocystic & 1 & 0.4 \\
\hline Adenosquamous low grade & 1 & 0.4 \\
\hline Apocrine & 1 & 0.4 \\
\hline \multicolumn{3}{|l|}{ Grade } \\
\hline G1 & 1 & 0.4 \\
\hline $\mathrm{G} 2$ & 75 & 32.3 \\
\hline $\mathrm{G} 3$ & 145 & 62.5 \\
\hline Non-specified & 11 & 4.7 \\
\hline \multicolumn{3}{|l|}{ TSR (Tumor stroma ratio) } \\
\hline Stroma high (>50\%) & 100 & 43.1 \\
\hline Stroma low $(<=50 \%)$ & 132 & 56.9 \\
\hline \multicolumn{3}{|l|}{ Ki-67 } \\
\hline$=<15 \%$ & 12 & 5.2 \\
\hline$>16 \%-=<30 \%$ & 39 & 16.8 \\
\hline$>30 \%$ & 130 & 56 \\
\hline Non-specified & 51 & 22 \\
\hline \multicolumn{3}{|l|}{ Necrosis } \\
\hline Yes & 85 & 36.6 \\
\hline No & 147 & 63.4 \\
\hline \multicolumn{3}{|l|}{ DCIS (LCIS) } \\
\hline Yes & 14 & 6.1 \\
\hline No & 218 & 93.9 \\
\hline \multicolumn{3}{|l|}{ Surgery } \\
\hline Breast preserving surgery & 141 & 60.8 \\
\hline Mastectomy & 91 & 39.2 \\
\hline \multicolumn{3}{|l|}{ Adjuvant chemotherapy } \\
\hline Yes & 207 & 89.2 \\
\hline No & 25 & 10.8 \\
\hline \multicolumn{3}{|l|}{ Radiotherapy } \\
\hline Yes & 203 & 87.5 \\
\hline No & 29 & 12.5 \\
\hline
\end{tabular}

operational material so biopsies and operational material after chemotherapy were excluded with eventual number of 232 samples. Tumor stage was evaluated based of the Seventh Edition of the TNM Classification of Malignant Tumors [15]. Stage IV tumors and patients with another type of malignancy were excluded. Triple negative breast cancer was defined according to the negative immunohistochemical expression of estrogen receptor (Clone EP1, Dako, USA), progesterone receptor (Clone PgR 636, Dako, USA) and HER2 (Clone SP3, Thermoscientific, USA). Overall survival (OS) was defined as the duration from the date of diagnosis to death from any cause or last follow-up. Disease-free survival (DFS) was defined as the duration of time from the date of diagnosis to locoregional or distant recurrence. Study protocol complied with the Declaration of Helsinki and was approved by National Medical Bogomolets University Ethics Committee (№5\24.01.2018).

Tumor stroma ratio was evaluated according to protocol proposed by Mesker et al. for establishment of TSR in colon cancer [16]. The most invasive part of the tumor was selected. Then $5 \mathrm{x}$ magnification was used to search for the area which contains the biggest amount of stroma. This area was zoomed using 10x magnification and the most stromal area was selected again. Tumor cells were presented on all sides of the view. Finally, stroma amount was reported using 10\% increments. According to the results patients were categorized into stroma-low group $(\leq 50 \%)$ and stroma-high group $(>50 \%)$.

All samples were analyzed by two pathologists who were not informed about clinical information of the patients.

Additional histopathological parameters which were evaluated included nuclear grade, histological type, presence of necrosis, presence of ductal or lobular carcinoma in situ (DCIS or LCIS) and Ki-67 (table I).

Statistical analysis was performed using EZR 1.35 software package ( $\mathrm{R}$ statistical software version 3.4.3, $\mathrm{R}$ Foundation for Statistical Computing, Vienna, Austria) [17]. The Cohen's Kappa coefficient was used to calculate inter-observer variability. The Kaplan-Meier method with logrank test was used to perform the survival curves. The Cox proportional hazards model was used to calculate univariate and multivariate hazard ratios (HR) for the parameters with $95 \%$ confidence interval (CI). Akaike information criterion (AIC) was used for selection of minimal set of parameters for multivariate analysis. p-Values of less than 0.05 were considered significant. Cutoff values for TSR had been chosen before statistical analysis was performed.

\section{RESULTS AND DISCUSSION}

350 patients were initially included in the study. However, only operational material without previous chemotherapy was taken into study. Patients who had biopsy and treatment options preliminary $(\mathrm{n}=89)$ were excluded. From 261 samples left some $(n=29)$ contained too less of invasive component or there was lack of clinical information so they were excluded. Totally 232 patients were included. A total of 51 patients died during the study, 72 patients had 


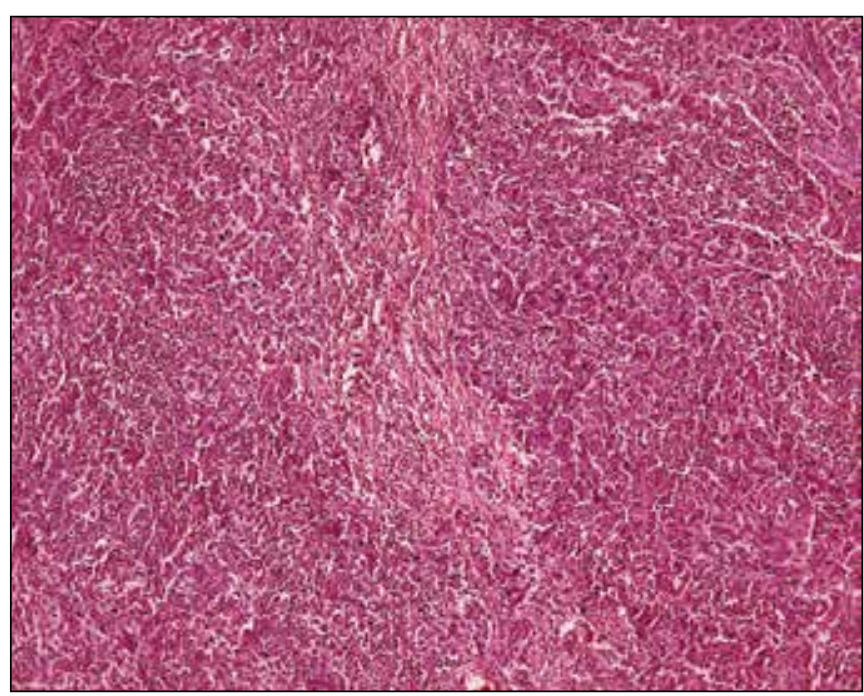

Fig 1. Stroma low (10\%) invasive breast carcinoma. H\&E, x10.

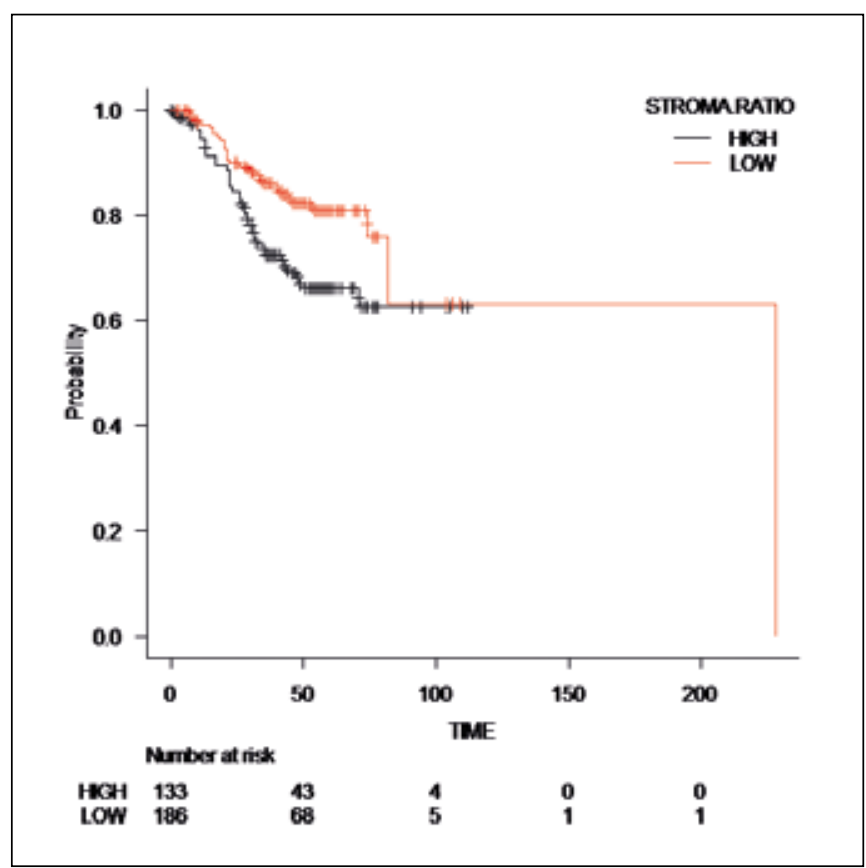

Fig.3.Kaplan-Meier curve for overall survival of patients stratified by tumor stroma ratio. $\mathrm{P}=0.008$

recurrent disease. The median follow-up period was 3.8 years (range from 0.3 to 9.3) for OS and 3.2 (range from 0.2 to 9.3 ) for DFS.

Tumors were categorized as stroma high $(\mathrm{n}=100)$ and stroma low $(\mathrm{n}=132)$ (figs 1,2$)$.

The three-year OS rate was $86.9 \pm 2.6 \%$ for low TSR group and $75.3 \pm 3.9 \%$ for high TSR group. The three-year DFS rate was $75.6 \pm 3.32 \%$ for low TSR group and $61.3 \pm 4.54 \%$ for high TSR group (Fig.3,4).

Kaplan-Meier curves statistically significantly showed relationship between TSR and overall and disease-free survival rates. Univariate analysis showed that stroma high tumors had statistically significantly worse OS (HR $1.89 ; 95 \%$ CI 1.18-3.03, $\mathrm{p}=0.008$ ) and DFS (HR 1.55; 95\% CI 1.05-2.28, $\mathrm{p}=0.027$ ) (tables II, III).

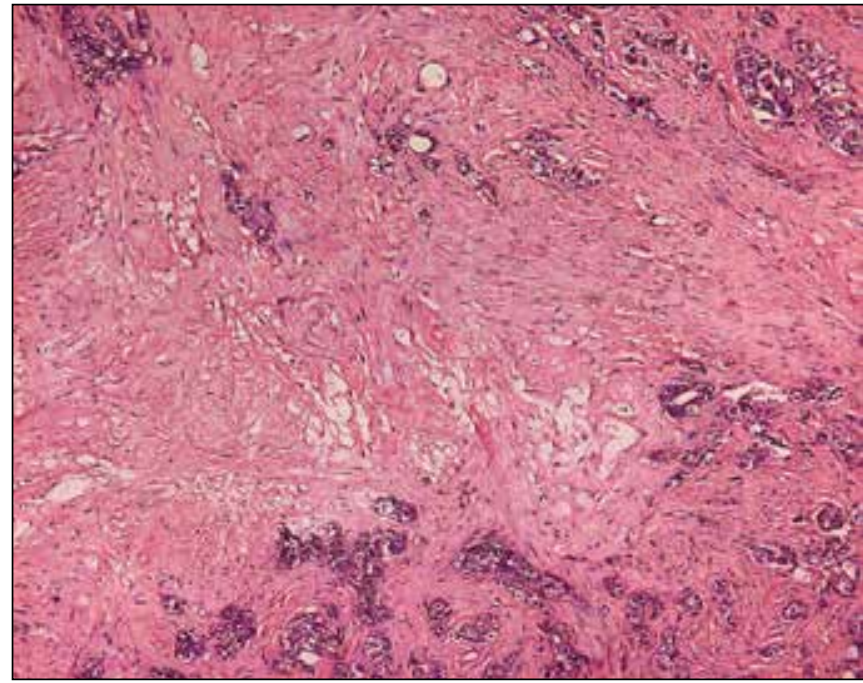

Fig 2. Stroma high (80\%) invasive breast carcinoma. H\&E, x10.

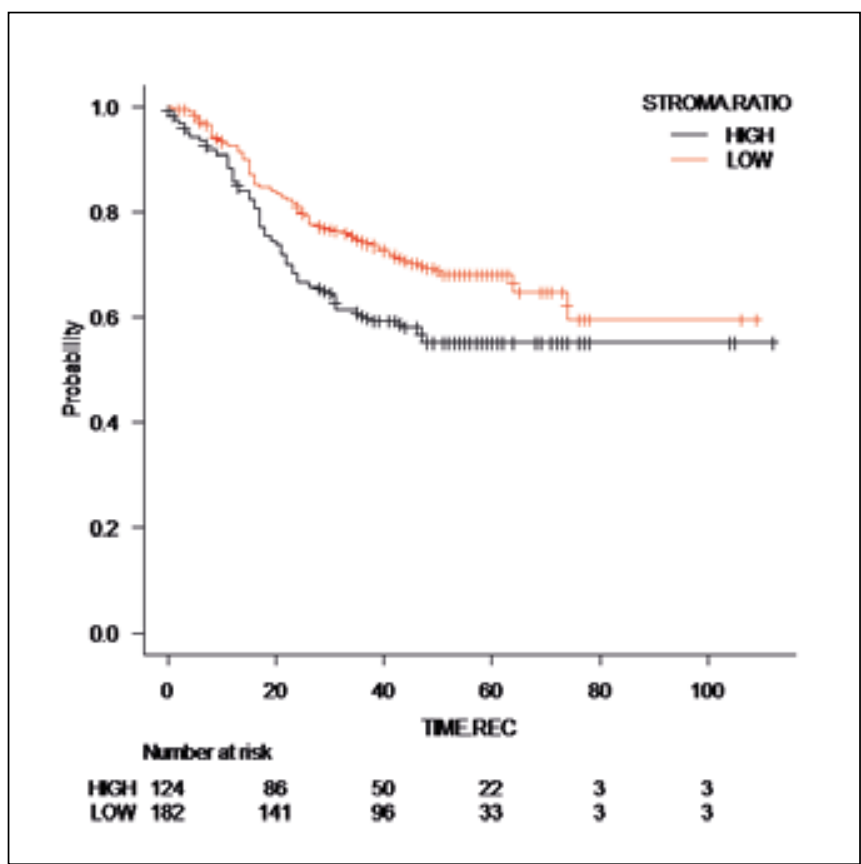

Fig 4. Kaplan-Meier curve for disease-free survival stratified by tumor stroma ratio. $\mathrm{P}=0.03$

After stepwise selection tumor size ( $\mathrm{pT}$ ), regional lymph nodes $(\mathrm{pN})$ and tumor stroma ratio (TSR) were highlighted as parameters statistically significantly connected both to overall and disease-free survival (table IV, V).

TSR is an independent prognostic factor both in univariate and multivariate Cox regression analysis for OS and DFS.

TSR was assessed by two pathologists. In 25 cases (7.8\%) there was no agreement in TSR at first individual assessment with kappa coefficient 0.84 which indicates overall good agreement. Kappa coefficient for TILS was 0.71, indicating good agreement as well. Second evaluation of debatable slides allowed to find consensus.

Tumor microenvironment which consist from cancer-associated fibroblasts, endothelial cells, pericytes and immune cell 
Table 2. Univariate Cox regression analysis of factors predicting overall survival in triple negative breast cancer

\begin{tabular}{|c|c|c|c|}
\hline \multirow[t]{2}{*}{ Parameter } & \multicolumn{3}{|c|}{ Univariate analysis } \\
\hline & Hazard ratio & $\begin{array}{l}\text { 95\% confidence } \\
\text { interval }\end{array}$ & $P$-value \\
\hline Age & & & 0.580 \\
\hline \multicolumn{4}{|l|}{$<40$} \\
\hline $40-60$ & 1.2 & $0.47-3.04$ & 0.705 \\
\hline $60>$ & 1.47 & $0.57-3.75$ & 0.425 \\
\hline \multicolumn{4}{|l|}{ Grade } \\
\hline \multicolumn{4}{|l|}{ Grade III } \\
\hline Grade II & 1.09 & $0.66-1.81$ & 0.738 \\
\hline Histological type & & & 0.321 \\
\hline \multicolumn{4}{|l|}{ Lobular } \\
\hline NST & 0.517 & $0.22-1.20 .000$ & 0.124 \\
\hline Carcinomas with medullary features & 0.000 & $0.26-4.2$ & 0.995 \\
\hline Papillary carcinoma & 1.049 & $0.25-6.06$ & 0.946 \\
\hline Others & 1.219 & & 0.808 \\
\hline Primary tumor & & & 0.0006 \\
\hline \multicolumn{4}{|l|}{$\mathrm{T} 1$} \\
\hline $\mathrm{T} 2$ & 1.48 & $0.73-2.37$ & 0.179 \\
\hline T3 & 4.4 & $2.04-11.36$ & 0.0006 \\
\hline $\mathrm{T} 4$ & 3.4 & $3.26-7.44$ & 0.002 \\
\hline Regional lymph nodes & & & 0.003 \\
\hline \multicolumn{4}{|l|}{ NO } \\
\hline N1 & 1.68 & $0.96-2.92$ & 0.066 \\
\hline N2 & 2.9 & $1.6-5.27$ & 0.0004 \\
\hline N3 & 3.038 & $1.33-6.93$ & 0.008 \\
\hline \multicolumn{4}{|l|}{ Tumor stroma ratio } \\
\hline \multicolumn{4}{|l|}{ High } \\
\hline Low & 1.89 & $1.18-3.03$ & 0.008 \\
\hline
\end{tabular}

Table 3. Univariate Cox regression analysis of factors predicting disease-free survival in triple negative breast cancer

\begin{tabular}{|c|c|c|c|}
\hline \multirow[t]{2}{*}{ Parameter } & \multicolumn{3}{|c|}{ Univariate analysis } \\
\hline & Hazard ratio & $\begin{array}{l}\text { 95\% confidence } \\
\text { interval }\end{array}$ & P -value \\
\hline Age & & & 0.097 \\
\hline \multicolumn{4}{|l|}{$<40$} \\
\hline $40-60$ & 0.9 & $0.38-2.14$ & 0.827 \\
\hline $60>$ & 1.41 & $0.61-3.28$ & 0.428 \\
\hline \multicolumn{4}{|l|}{ Grade } \\
\hline \multicolumn{4}{|l|}{ Grade III } \\
\hline Grade II & 0.98 & $0.65-1.47$ & 0.916 \\
\hline Histological type & & & 0.637 \\
\hline \multicolumn{4}{|l|}{ Lobular } \\
\hline NST & 0.613 & $0.28-1.33$ & 0.213 \\
\hline Carcinomas with medullary features & 0.000 & 0.000 & 0.994 \\
\hline Papillary carcinoma & 1.049 & $0.26-4.2$ & 0.732 \\
\hline Others & 1.102 & $0.28-4.26$ & 0.888 \\
\hline Primary tumor & & & 0.000 \\
\hline \multicolumn{4}{|l|}{$\mathrm{T} 1$} \\
\hline $\mathrm{T} 2$ & 1.14 & $0.71-1.82$ & 0.591 \\
\hline T3 & 5.75 & $2.93-11.3$ & 0.000 \\
\hline $\mathrm{T} 4$ & 2.28 & $1.12-4.64$ & 0.023 \\
\hline Regional lymph nodes & & & 0.000 \\
\hline \multicolumn{4}{|l|}{ NO } \\
\hline N1 & 1.62 & $1.00-2.61$ & 0.049 \\
\hline N2 & 2.43 & $1.41-4.18$ & 0.001 \\
\hline N3 & 2.39 & $1.10-5.00$ & 0.026 \\
\hline \multicolumn{4}{|l|}{ Tumor stroma ratio } \\
\hline \multicolumn{4}{|l|}{ Low } \\
\hline High & 1.55 & $1.05-2.28$ & 0.027 \\
\hline
\end{tabular}


Table 4. Multivariate Cox regression analysis of factors predicting overall survival in triple negative breast cancer with stepwise selection based on AIC

\begin{tabular}{cccc}
\hline Parameter & \multicolumn{3}{c}{ Multivariate analysis } \\
& Hazard ratio & $\begin{array}{c}\text { 95\% confidence } \\
\text { interval }\end{array}$ & P value \\
\hline Primary tumor (pT) & & & 0.036 \\
T1 & 1.24 & $0.68-2.27$ & 0.292 \\
T2 & 3.80 & $1.45-9.95$ & 0.006 \\
T3 & 2.09 & $0.83-5.24$ & 0.113 \\
T4 & & & 0.035 \\
N0 & & & \\
N1 & 1.24 & $0.64-2.39$ & 0.064 \\
N2 & 2.16 & $1.05-4.45$ & 0.058 \\
N3 & 3.47 & $1.46-8.23$ & 0.002 \\
\hline Tumor stroma ratio & & & \\
Low & & & 0.002 \\
High & 2.11 & $1.29-3.43$ &
\end{tabular}

Table 5. Multivariate Cox regression analysis of factors predicting DFS in triple negative breast cancer with stepwise selection based on AIC

\begin{tabular}{cccc}
\hline Parameter & \multicolumn{3}{c}{ Multivariate analysis } \\
& Hazard ratio & $\begin{array}{c}\text { 95\% confidence } \\
\text { interval }\end{array}$ & P value \\
\hline Primary tumor (pT) & & & 0.000 \\
T1 & 1.002 & $0.62-1.64$ & 0.986 \\
T2 & 4.63 & $2.15-10.03$ & 0.000 \\
T3 & 1.05 & $0.44-2.48$ & 0.915 \\
T4 & & & 0.003 \\
Regional lymph nodes (pN) & 1.31 & $0.76-2.26$ & 0.331 \\
N1 & 2.32 & $1.25-4.32$ & 0.007 \\
N2 & 2.82 & $1.28-6.20$ & 0.010 \\
N3 & & & \\
\hline Low & & & 0.004 \\
\hline High & 1.83 & $1.22-2.78$ &
\end{tabular}

infiltration significantly influences on tumor invasion ability and potential for metastasis [18]. Stroma as a part of the TME can also be responsible for chemotherapy resistance and treatment failures as well as be an object of targeted therapy [19, 20]. According to Mierke et al. [21] the impact of the tumor stroma on cancer progression is controversial as there are two different and opposing effects within the stroma. On one hand, it can promote and enhance the proliferation, survival and migration of cancer cells as a result of increased stroma rigidity. On the other hand, the stroma acts as a "steric obstacle" for cancer cell motility in dense three-dimensional extracellular matrices, when the pore size is smaller than the cell's nucleus.

Our study indicated that the tumor stroma ratio is an independent prognostic factor for triple negative breast cancer patients. Patients with high tumor stroma ratio statistically significantly show worse overall and disease-free survival compared to low tumor stroma ratio patients. Our results confirm results found in other studies investigating TSR in breast cancer patients.

Several studies show that biology of tumor associated stromal cells differs from their normal counterparts. In particular, fibroblasts found in tumor stroma and named as cancer-associated fibroblasts (CAFs) are distinct from normal ones in their "activated phenotype" with enhanced production of collagen and growth factors [22]. They have more rapid proliferation rate and can promote breast cancer invasion and proliferation [23]. They also have another from normal fibroblasts phenotype. According to reverse Warburg effect, described by Pavlidus et al. [24], CAFs undergo myofibroblastic differentiation and secrete lactate and pyruvate. Epithelial cancer cells can take up these energy-rich metabolites and use them in the Krebs cycle, thereby promoting efficient energy production, resulting again in a higher proliferative capacity.

Mesenchymal stem cells (MSCs) which migrate toward tumor stroma also may change their functions. They incorporate into TME and become cancer-assosiated (CA-MSCs) and also contribute to tumor progression by different interaction with tumor cells. It has been shown that cross-talk between tumour cells and MSCs increases metastatic potential and promote epithelial-to-mesenchymal transition [25]. Previously MSCs have been reported 
to have antitumor protective function including inhibition of angiogenesis, induction of tumor cell apoptosis and enhancement of immune response, but these effects are observed only when MSCs are used in higher ratios to tumor cells. The function of these cells is tissue dependent and naive MSC with antitumor effects can develop into CA-MSCs with pro-tumorigenic function. [26]

Still it is questionable whether biopsy is representative for TSR assessment. In our study only previously untreated operational material was investigated with concern that core biopsy can decrease opportunity for representative areas to be chosen. Study on esophageal adenocarcinomas [27] showed good reproducibility of tumor stroma ratio scoring in biopsies compared to operational material. But chemotherapy regimens are usually performed between biopsy and operational material in breast cancer patients obstructing such comparison.

Investigation of TSR doesn't require any additional costs and slide preparation and it is quite simple in methodology, that's why it can be easily added to routine breast cancer investigation. It can clarify prognosis of patients and probably expand treatment options in the future, that's why prospective cohort studies are needed to confirm eventually TSR value.

Our study has several limitations. Firstly, it is retrospective with prolonged time interval assessment (2009-2018) during which some treatment protocols have changed. Secondly, different chemotherapeutic agents were used, including regimens with taxanes, anthracyclins and platinum agents.

\section{CONCLUSION}

Tumor microenvironment influences tumor progression. Tumor stroma ratio can be easily evaluated on H\&E. Tumor stroma ratio is a strong independent prognostic marker for triple negative breast cancer with worse overall and disease-free survival for tumors that contain much stroma.

\section{REFERENCES}

1. Grybach S., Polishchuk L., Chekhun V. Analysis of the survival of patients with breast cancer depending on age, molecular subtype of tumor and metabolic syndrome. Exp Oncol. 2018; 40 (3): 243-248.

2. Gonçalves H.Jr., Guerra M.R., Duarte Cintra J.R. et al. Survival Study of Triple-Negative and Non-Triple-Negative Breast Cancer in a Brazilian Cohort. Clin Med Insights Oncol. 2018; 12:1-10.

3. Wahba H.A., El-Hadaad H.A. Current approaches in treatment of triplenegative breast cancer. Cancer Biol Med. 2015;12(2):106-116.

4. Su S., Chen J., Yao H. et al. CD10(+)GPR77(+)Cancer-Associated Fibroblasts Promote Cancer Formation and Chemoresistance by Sustaining Cancer Stemness. Cell. 2018;172(4):841-856.

5. Wang K., Ma W., Wang J. et al. Tumor-stroma ratio is an independent predictor for survival in esophageal squamous cell carcinoma. J Thorac Oncol. 2012;7(9):1457-61.

6. Mesker W.E., Liefers G.J., Junggeburt J.M. et al. Presence of a high amount of stroma and downregulation of SMAD4 predict for worse survival for stage I-II colon cancer patients. Cell Oncol. 2009;31(3):169-78.
7. Hale M.D., Hayden J.D., Grabsch H.I. Tumour-microenvironment interactions: role of tumour stroma and proteins produced by cancerassociated fibroblasts in chemotherapy response. Cell Oncol (Dordr). 2013;36(2):95-112.

8. Provenzano P.P., Cuevas C., Chang A.E. et al. Enzymatic targeting of the stroma ablates physical barriers to treatment of pancreatic ductal adenocarcinoma. Cancer Cell. 2012;21(3):418-429.

9. DekkerT.J., van de VeldeC.J., van Pelt G.W. et al. Prognostic significance of the tumor-stroma ratio:validation study in node-negative premenopausal breast cancer patients from the EORTC perioperative chemotherapy (POP) trial (10854). Breast Cancer Res Treat. 2013; 139(2):371-9.

10. Roeke T., Sobral-Leite M., Dekker T.J. et al. The prognostic value of the tumour-stroma ratio in primary operable invasive cancer of the breast: a validation study. Breast Cancer Res Treat. 2017;166(2):435-445.

11. Moorman A.M., Vink R., Heijmans H.J. et al. The prognostic value of tumour-stroma ratio in triple-negative breast cancer. Eur J Surg Oncol. 2012; 38(4):307-13.

12. Gujam F.J., Edwards J., Mohammed Z.M. et al. The relationship between the tumour stroma percentage, clinicopathological characteristics and outcome in patients with operable ductal breast cancer. $\mathrm{Br} J$ Cancer. 2014; 111(1):157-165.

13. Kramer C.J., Vangangelt K.M., van Pelt G.W. et al. The prognostic value of tumour-stroma ratio in primary breast cancer with special attention to triple-negative tumours: a review. Breast Cancer Res Treat. 2019;173(1):55-64.

14. Vangangelt K.M.H., Green A.R., Heemskerk I.M.F. et al. The prognostic value of the tumor-stroma ratio is most discriminative in patients with grade III or triple-negative breast cancer. Int I Cancer. 2020; 146(8):2296-2304.

15. Greene F.L., Sobin L.H. A worldwide approach to the TNM staging system: collaborative efforts of the AJCC and UICC.J Surg Oncol. 2009;99:269-272.

16. Mesker W.E., Junggeburt J.M., Szuhai K. et al. The carcinoma-stromal ratio of colon carcinoma is an independent factor for survival compared to lymph node status and tumor stage. Cell Oncol. 2007;29(5):387-98.

17. Kanda Y. Investigation of the freely available easy-to-use software 'EZR' for medical statistics. Bone Marrow Transplant. 2013; 48:452-458.

18. Khamis Z.I., Sahab Z.J., Sang Q.X. Active roles of tumor stroma in breast cancer metastasis. Int J Breast Cancer. 2012:574025. doi:10.1155/2012/574025.

19. Chen X., Song E. Turning foes to friends: targeting cancer-associated fibroblasts. Nat Rev Drug Discov. 2019; 18: 99-115.

20. Plava J., Cihova M., Burikova M. et al. Recent advances in understanding tumor stroma-mediated chemoresistance in breast cancer. Mol Cancer. 2019;18:67.

21. Mierke C.T., Sauer F., Grosser S. et al. The two faces of enhanced stroma: Stroma acts as a tumor promoter and a steric obstacle. NMR in Biomedicine. 2017. e3831. doi:10.1002/nbm.3831.

22. Raffaghello L., Dazzi F. Classification and biology of tumour associated stromal cells. Immunology Letters.2015; 168(2): 175182. doi:10.1016/j.imlet.2015.06.016.

23. Eiro N., González L., Martínez-Ordoñez A. et al. Cancer-associated fibroblasts affect breast cancer cell gene expression, invasion and angiogenesis. Cell Oncol (Dordr). 2018;41(4):369-378. doi:10.1007/ s13402-018-0371-y.

24. Pavlides S., Whitaker-Menezes D., Castello-Cros R. et al. The reverse Warburg effect: aerobic glycolysis in cancer associated fibroblasts and the tumor stroma. Cell Cycle. 2009;8(23):3984-4001. doi:10.4161/ cc.8.23.10238. 
25. Ahn S.Y. The Role of MSCs in the Tumor Microenvironment and Tumor Progression. Anticancer Res. 2020;40(6):3039-3047. doi:10.21873/ anticanres.14284.

26. Atiya H., Frisbie L., Pressimone C., Coffman L. Mesenchymal Stem Cells in the Tumor Microenvironment. Adv Exp Med Biol. 2020; 1234:31-42. doi:10.1007/978-3-030-37184-5_3.

27. Courrech Staal E.F., Smit V.T., van Velthuysen M.L. et al. Reproducibility and validation of tumour stroma ratio scoring on oesophageal adenocarcinoma biopsies. Eur J Cancer. 2011;47(3):375-382. doi:10.1016/j.ejca.2010.09.043.

The work was performed as a part of budget scientific research work "Development of histological and molecular-biological criteria for differential diagnosis of tumors and precancerous changes in organs and their prognostic value" (2018-2021, state registration number 0119U101131).

\section{ORCID and contributionship:}

Liubov M. Zakhartseva: 0000-0001-6838-9970 A,E,F

Mariia A. Yanovytska: 0000-0002-5919-081X ${ }^{B, C, D}$

\section{Conflict of interest:}

The Authors declare no conflict of interest.

\section{CORRESPONDING AUTHOR}

Mariia A. Yanovytska

Bogomolets National Medical University

69 Verhovynna str., 02000 Kyiv, Ukraine

tel: +380634714542

e-mail:m.yanovytskaya@gmail.com

Received: 02.10 .2020

Accepted: 01.03.2021

\footnotetext{
A - Work concept and design, B - Data collection and analysis, C - Responsibility for statistical analysis,
}

D-Writing the article, $\mathbf{E}$-Critical review, $\mathbf{F}$ - Final approval of the article 